\title{
Drip and ship: Una práctica usual en el tratamiento de ictus isquémico por oclusión de arterias proximales en países de altos ingresos pero anecdótica en Colombia
}

\section{Drip and ship: a prevalent practice in acute stroke with large vessel occlusion in high- income countries but anecdotal in Colombia}

Pablo Amaya,,2 Akemi Arango, ${ }^{3}$ Carlos Pardo, Natalia Llanos-Leyton, ${ }_{1}^{1}$ Edgar Folleco,, Hernán Mauricio Patiño-Rodríguez, Ángel Basilio Corredor-Quintero ${ }^{6}$

\section{Resumen}

El Drip and Ship consiste en administrar trombolisis endovenosa en centro primario y remisión para trombectomía mecánica.

Objetivo: Describir la experiencia Drip and Ship como intervención del ictus isquémico en dos regiones de Colombia.

Métodos: Serie de casos retrospectiva de pacientes tratados con Drip and Ship durante 2019 y primer cuartil de 2021. Se compararon variables como puerta-imagen, puerta-aguja, puerta-ingle y desenlaces clínicos. Se calcularon medidas de tendencia central y estadísticas descriptivas.

Resultados: Se registraron seis pacientes con ictus isquémico y oclusión proximal. Mediana de 60 minutos de inicio de síntomas, puerta-aguja 75 minutos, tiempo door-in door-out 167.5 minutos, tiempo de traslado 91.5 minutos para remisión desde otra ciudad. Cinco pacientes recibieron trombectomía mecánica con TICI 2c-3. 50\% de los pacientes con Rankin modificado favorable al egreso y $84 \%$ a tres meses.

Conclusiones: En el registro RES-Q entre 2019 y el primer cuartil de 2021, se consignaron 5954 casos de ictus isquémico en Colombia, 20 pacientes tratados con Drip and Ship (0,33\%). Los tiempos de puerta- aguja y de remisión fueron mayores a lo recomendado en guías internacionales, siendo mayor en los pacientes provenientes de otras ciudades. Se requiere estructurar redes de atención para mejorar tiempos de tratamiento y número de pacientes tratados.

Palabras clave: Ictus isquémico, trombectomía, trombólisis, país de medianos ingresos

\section{Abstract}

The Drip and Ship consists of giving intravenous thrombolysis in the primary center and referral for mechanical thrombectomy

Objective: To describe the experience of Drip and Ship in acute ischemic stroke in two regions of Colombia.

Methods: We conducted a retrospective case series study of patients undergoing Drip and Ship from 2019 to first quartile of 2021. We compared times: door-to-image, door-to-needle, door-to-groin and clinical outcomes. Measures of central tendency and descriptive statistics were calculated.

Results: Six patients with ischemic stroke and large vessel occlusion were registered. Median of 60 minutes of the onset of symptoms, 75 minutes for door-to-needle, 167.5 minutes for door-in door-out time, and 91.5 minutes for travel time referred from another city. Five patients received mechanical thrombectomy with TICI 2c-3. 50\% of patients with favorable modified Rankin scale at discharge and $84 \%$ at three months.

Conclusions: According to the RES-Q registry, between 2019 and the first quartile of 2021, there were 5954 cases of ischemic stroke cases in Colombia, and 20 patients received Drip and Ship strategy (0.33\%). In agreement to international guidelines, door-toneedle and remission times were longer than recommended, especially in patients from other cities. It is necessary to structure care networks to improve treatment times and the number of patients treated.

Keywords: Ischemic stroke, thrombectomy, thrombolysis, middle income countries

Rev. Ecuat. Neurol. Vol. 30, Nº 3, 2021

'Universidad Icesi, Facultad de Ciencias de la Salud, Departamento de Neurología, Calle 18 No. 122-135, Cali, Colombia

${ }^{2}$ Fundación Valle del Lili, Programa ACV, Departamento de Neurología, Cra 98 No 18-49, Cali 760032, Colombia.

${ }^{3}$ Centro de Investigaciones Clínicas (CIC), Fundación Valle del Lili, Cali. Cra 98

No 18-49, Cali 760032, Colombia

${ }^{4}$ Fundación Valle del Lili, Departamento de Radiología, Cra 98 No 18-49, Cali

760032, Colombia.

${ }^{5}$ Departamento de Neurología y Neurointervencionismo, Hospital

Universitario Infantil de San José, Bogotá, Colombia .

${ }^{6}$ Departamento de Neurología, Hospital Departamental Universitario del

Quindío San Juan de Dios, Armenia, Colombia.
Correspondencia:

Pablo Amaya, MD.

Fundación Valle del Lili, Cra 98 No 18-49, Cali 760032, Colombia.

Teléfono: 3319090 Ext. 4022.

E-mail: pablo.ricardo@fvl.org.co 


\section{Introducción}

Se conoce como "Drip and Ship" al proceso de tratamiento con trombólisis endovenosa en un centro primario y posteriormente el traslado a un centro subespecializado en ictus para trombectomía mecánica. Esta estrategia busca ofrecer tratamiento endovascular a pacientes de áreas distantes a centros de alta complejidad donde los especialistas y recursos, como salas de angiografías, son limitados. En países de medianos ingresos como Colombia se cuenta con una escasa cantidad de centros de trombectomía, concentrados en ciudades capitales, limitando el acceso a tratamiento agudo del ictus isquémico a los pacientes de ciudades pequeñas o zonas rurales. En Colombia, solo 13 ciudades de los 1103 municipios cuentan con centros de trombectomía y de los 34 centros habilitados para realizar esta intervención, solo 14 de ellos prestan servicios las 24 horas los 7 días de la semana. ${ }^{3}$

Ensayos clínicos controlados han demostrado que la combinación de trombolisis endovenosa con rt-PA (TE) y trombectomía mecánica (TM) es efectiva en los pacientes con ictus isquémico con oclusión de vaso proximal. ${ }^{4}$ Las guías internacionales recomiendan la administración de alteplasa en pacientes en los que se considera la realización de (TM) en aquellos con tiempos de ventana (tiempo desde el inicio de los síntomas hasta la llegada al centro primario) de 0 a 4,5 horas y adicionalmente el uso de stent retriever o trombectomía de aspiración directa. Para aquellos pacientes con tiempos de ventana de 6 a 16 horas con oclusión de vaso proximal y que cumplan otros criterios de elegibilidad DAWN u DEFUSE 3, se recomienda TM. En pacientes con tiempos de ventana de 16 a 24 horas con oclusión de arteria proximal, y que cumplan otros criterios DAWN, la trombectomía es razonable.

La estrategia "Drip and Ship" en el marco de una red de ictus isquémico que incluya el uso de telemedicina aumenta el uso de rt-PA en centros de primer nivel y optimiza la selección de pacientes para transferencia a centros sub-especializados de manejo de ictus isquémico, logrando mejores desenlaces. ${ }^{6}$

A continuación se presentan seis casos de Drip and Ship realizados en dos centros sub-especializados en ictus isquémico del centro y suroccidente de Colombia remitidos de centros primarios sin disponibilidad de trombectomía. Adicionalmente, se hace una revisión narrativa sobre esta modalidad de tratamiento y las barreras de acceso a terapia endovascular en nuestro país.

\section{Métodos}

Se realizó una recolección retrospectiva, multicéntrica de una serie de casos de pacientes que recibieron trombolisis con alteplasa a dosis de $0,9 \mathrm{mg} / \mathrm{kg}$ y fueron remitidos para trombectomía mecánica en un centro subespecializado. La búsqueda de los casos se realizó a través de una convocatoria del comité vascular de la Asociación Colombiana de Neurología a los centros con programa de ictus y capacidad de trombectomía mecánica durante el periodo de tiempo comprendido entre 2019 y el primer cuartil del año 2021. Adicionalmente, se revisaron los datos entregados en la estadística nacional de los centros participantes en el Registry of Stroke Care Quality (RESQ), un registro internacional multicéntrico financiado por la European Stroke Organization y avalado por la World Stroke Organization de calidad de la atención, que busca mejorar el sistema de atención de ictus, para identificar los casos señalados en el país que se sometieron a la estrategia Drip and Ship. Se obtuvieron los datos en cada centro del registro institucional de ictus isquémico previamente aprobado por el comité de ética local y se incluyeron los casos de los centros que respondieron a la convocatoria, contaran con diligenciamiento completo del formato de recolección de datos y desearan participar en el desarrollo del manuscrito.

Se incluyeron variables demográficas como: edad, sexo, antecedentes patológicos, factores de riesgo cardiovascular como hipertensión arterial, diabetes, dislipidemia, fibrilación auricular. Se incluyó el registro de las variables de evolución y atención, tiempo desde inicio de los síntomas hasta el ingreso a urgencias, el tiempo transcurrido en minutos desde el ingreso del paciente hasta la administración de trombolítico (puerta-aguja), tiempo de tratamiento en centro primario (door in - door out), tiempo de traslado y tiempo transcurrido en minutos desde llegada al centro sub-especializado hasta el momento de inicio de la intervención endovascular (puerta-ingle). Mediante la escala National Institutes of Health Stroke Scale (NIHSS) se evaluó la severidad del ictus ( 0 - 42 , puntajes más elevados indican déficit neurológico más severo) y desenlace funcional con la escala modificada de Rankin (mRS). Con la escala de Alberta Stroke Program Early CT Score (ASPECTS) se evaluaron los cambios isquémicos evidenciados en neuroimágenes como tomografía computarizada (TC) de cráneo simple (no contrastada). Se realizó TC de cráneo simple a todos los pacientes en el centro primario y en el centro avanzado. El grado de recanalización post trombectomía, se midió basado en la escala de Thrombolysis In Cerebral Infarction (TICI). Para el análisis estadístico se usó el programa SPSS versión 21 (IBM Corporation E.E.U.U), se calcularon las medidas de tendencia central y estadísticos descriptivos, para reportar las principales características demográficas de los pacientes y los tiempos de atención incluyendo tiempo de inicio a puerta de síntomas. 
Tabla 1. Características clínicas de los pacientes.

\begin{tabular}{|c|c|c|c|c|c|c|}
\hline & Caso 1 & Caso 2 & Caso 3 & Caso 4 & Caso 5 & Caso 6 \\
\hline Procedencia & Armenia & Popayán & Bogotá & Bogotá & Bogotá & Bogotá \\
\hline $\begin{array}{l}\text { Distancia recorrida desde centro } \\
\text { primario a centro integral }(\mathrm{Km})\end{array}$ & 192 & 141 & 9.9 & 9.9 & 8.6 & 8.6 \\
\hline Tiempo de traslado ( $\mathrm{min})$ & 175 & 182 & 80 & 60 & 45 & 60 \\
\hline Sexo & Femenina & Masculino & Femenina & Femenina & Femenina & Femenina \\
\hline Edad & 40 & 75 & 76 & 62 & 65 & 52 \\
\hline Antecedentes & ACV & $\begin{array}{l}\text { HTA, diabetes } \\
\text { mellitus, HPB }\end{array}$ & HTA & $\begin{array}{l}\text { FA no } \\
\text { anticoagulada }\end{array}$ & Ninguno & $\begin{array}{l}\text { FA no } \\
\text { anticoagulada }\end{array}$ \\
\hline mRS previo & 1 & 0 & 0 & 0 & 0 & 0 \\
\hline Cuadro Clínico & $\begin{array}{l}\text { Afasia y } \\
\text { hemiparesia } \\
\text { derecha }\end{array}$ & $\begin{array}{l}\text { Síncope, afasia y } \\
\text { monoparesia } \\
\text { derecha }\end{array}$ & $\begin{array}{l}\text { Afasia, desviación } \\
\text { forzada de la } \\
\text { mirada y paresia } \\
\text { fascio braquial }\end{array}$ & $\begin{array}{l}\text { Afasia y hemiplejia } \\
\text { derecha }\end{array}$ & $\begin{array}{l}\text { Afasia y hemiplejia } \\
\text { derecha }\end{array}$ & $\begin{array}{l}\text { Plejia Hemicuerpo } \\
\text { derecho }\end{array}$ \\
\hline NIHSS ingreso & 28 & 12 & 19 & 16 & 17 & 12 \\
\hline Ventana & 28 & 180 & 60 & 30 & 180 & - \\
\hline Imagen de remisión & TC simple & TC simple & CTA & CT & CTA & DWI/FLAIR \\
\hline $\begin{array}{l}\text { ASPECTS } \\
\text { centro primario }\end{array}$ & 10 & 10 & 10 & 10 & 10 & 8 \\
\hline Manejo agudo centro primario & Trombolisis IV & Trombolisis IV & Trombolisis IV & Trombolisis IV & Trombolisis IV & Trombolisis IV \\
\hline Tiempo puerta -aguja & 90 & 180 & 100 & 50 & 45 & 60 \\
\hline Tiempo door in door out (Min) & 135 & 340 & 200 & 100 & 120 & 300 \\
\hline NIHSS de ingreso centro integral & 16 & 19 & 19 & 16 & 12 & 12 \\
\hline Imagen centro integral & CTA & CTP & CT & CTA & CTP & CTP \\
\hline Arteria ocluida & ACMI porción M1 & ACMI porción M1 & ACMI porción M1 & ACMI porción M1 & ACMI porción M1 & ACMI porción M1 \\
\hline ASPECTS centro avanzado & 6 & 3 & 7 & 8 & 7 & 8 \\
\hline Trombectomía mecánica / TICI & Si / $2 \mathrm{C}$ & No/NA & $\mathrm{SI} / 3$ & $\mathrm{SI} / 3$ & $\mathrm{SI} / 3$ & $\mathrm{SI} / 3$ \\
\hline $\begin{array}{l}\text { Exámenes de extensión / } \\
\text { hallazgos relenvantes }\end{array}$ & $\begin{array}{l}\text { Eco TT: Foramen } \\
\text { oval permeable + } \\
\text { aneurisma septal }\end{array}$ & $\begin{array}{l}\text { EKG: fibrilación } \\
\text { auricular }\end{array}$ & $\begin{array}{l}\text { Eco-TT, Holter, } \\
\text { AngioTAC }\end{array}$ & $\begin{array}{l}\text { Eco-TT, Holter, } \\
\text { AngioTAC }\end{array}$ & $\begin{array}{l}\text { Eco-TT, Holter, } \\
\text { AngioTAC }\end{array}$ & $\begin{array}{l}\text { Eco-TT, Holter, } \\
\text { AngioTAC }\end{array}$ \\
\hline Etiología ACV & Cardioembólico & Cardioembólico & Cardioembólico & Cardioembólico & Desconocido & Cardioembólico \\
\hline Días de estancia hospitalaria & 5 & 4 & 5 & 6 & 7 & 6 \\
\hline mRS al egreso & 2 & 6 & 3 & 3 & 1 & 1 \\
\hline mRS a los 3 meses & 2 & 6 & 2 & 1 & 1 & 1 \\
\hline
\end{tabular}

HTA: Hipertensión arterial. HPB: hiperplasia prostática benigna. FA: Fibrilación auricular. ACM: Arteria cerebral media. Eco TT: Ecocardiograma transtorácico. EKG:

Electrocardiograma. NIHSS: escala National Institutes of Health Stroke Scale. mRS: modified rankin scale. Km: Kilómetros. Min: minutos. Kg: kilogramo. mg: miligramo. IV: intravenosa. ASPECTS: Alberta Stroke Program Early CT Score. TICI: Thrombolysis In Cerebral Infarction. TC: tomografía computarizada. CTA: Angiografía por tomografía computarizada. CTP: tomografía computarizada con perfusión. DWI: diffusion-weighted imaging. FLAIR: fluid-attenuated inversion recovery.

Tabla 2. Comparación de grupos. Grupo A: centros de diferentes ciudades (Cali-Popayán-Armenia) y grupo B: centros de 1 sola ciudad (Bogotá)

\begin{tabular}{|llll|}
\hline $\begin{array}{l}\text { Variable } \\
\text { mediana (Q1-Q3) }\end{array}$ & $\begin{array}{l}\text { GRUPO A } \\
(\mathbf{n = 2})\end{array}$ & $\begin{array}{l}\text { GRUPO B } \\
(\mathbf{n = 4})\end{array}$ & $\begin{array}{l}\text { TOTAL CASOS } \\
\text { (n=6) }\end{array}$ \\
\hline Edad en años & $57.5(48.75-66.25)$ & $63.5(59.5-67.75)$ & $63.5(54.5-72.5)$ \\
\hline Sexo femenino* & $1(50 \%)$ & $4(100 \%)$ & $5(83,5 \%)$ \\
\hline Comorbilidades* & $50 \%$ Antecedente de ACV & $25 \%$ HTA y 50\% FA sin anticoagulación & $60(30-180)$ \\
\hline Tiempo ventana en minutos & $104(66-142)$ & $60(45-120)$ & 10 \\
\hline ASPECTS en centro primario* & 10 & 10 & $9.9(153.75-179.25)$ \\
\hline Distancia recorrida en Km & $166.5(153.75-179.25)$ & $9.25(8.6-8.6)$ & $70(60-151.25)$ \\
\hline Tiempo de traslado en minutos & $178.5(176.75-180.25)$ & $60(56.25-65)$ & $75(52.5-97-5)$ \\
\hline Tiempo puerta-aguja en minutos & $135(112.5-157-5)$ & $55(48.75-70)$ & ACMI M1(100\%) \\
\hline Arteria ocluida* & $\mathrm{ACMI}$ M1 (100\%) & $\mathrm{ACMI} \mathrm{M1(100 \% )}$ & $7(6.25-7.75)$ \\
\hline ASPECTS en centro integral & $4.5(3.75-5.25)$ & $7.5(7-8)$ & $167.5(123.75-275)$ \\
\hline Tiempo door in door out en minutos & $237.5(186.25-288.75)$ & $160(115-225)$ & $5.5(5-6)$ \\
\hline Días de estancia hospitalaria & $4.5(4.25-4.75)$ & $6(5.75-6.25)$ & $2.5(1.25-3)$ \\
\hline mRs al egreso & $4(3-5)$ & $2(1-3)$ & $1.5(1-2)$ \\
\hline mRs a los 3 meses & $4(3-5)$ & $1(1-1.25)$ & \\
\hline *Promedio y DE & & & \\
\hline
\end{tabular}

HTA: Hipertensión arterial. FA: Fibrilación Auricular. ACV: Ataque cerebrovascular. Km: Kilómetros. ASPECTS: Alberta Stroke Program Early CT Score. mRS: modified rankin scale. 
Figura 1. Mapas de Colombia y Bogotá con distancias recorridas por los pacientes desde centro primario de ACV hasta el centro de trombectomía. Figura 1a. Recorrido desde centros primarios a centro de trombectomía en Cali. Figura $\mathbf{1 b .}$ Recorrido desde centro primario de ACV a centro de trombectomía en Bogotá. (fuente Google maps).

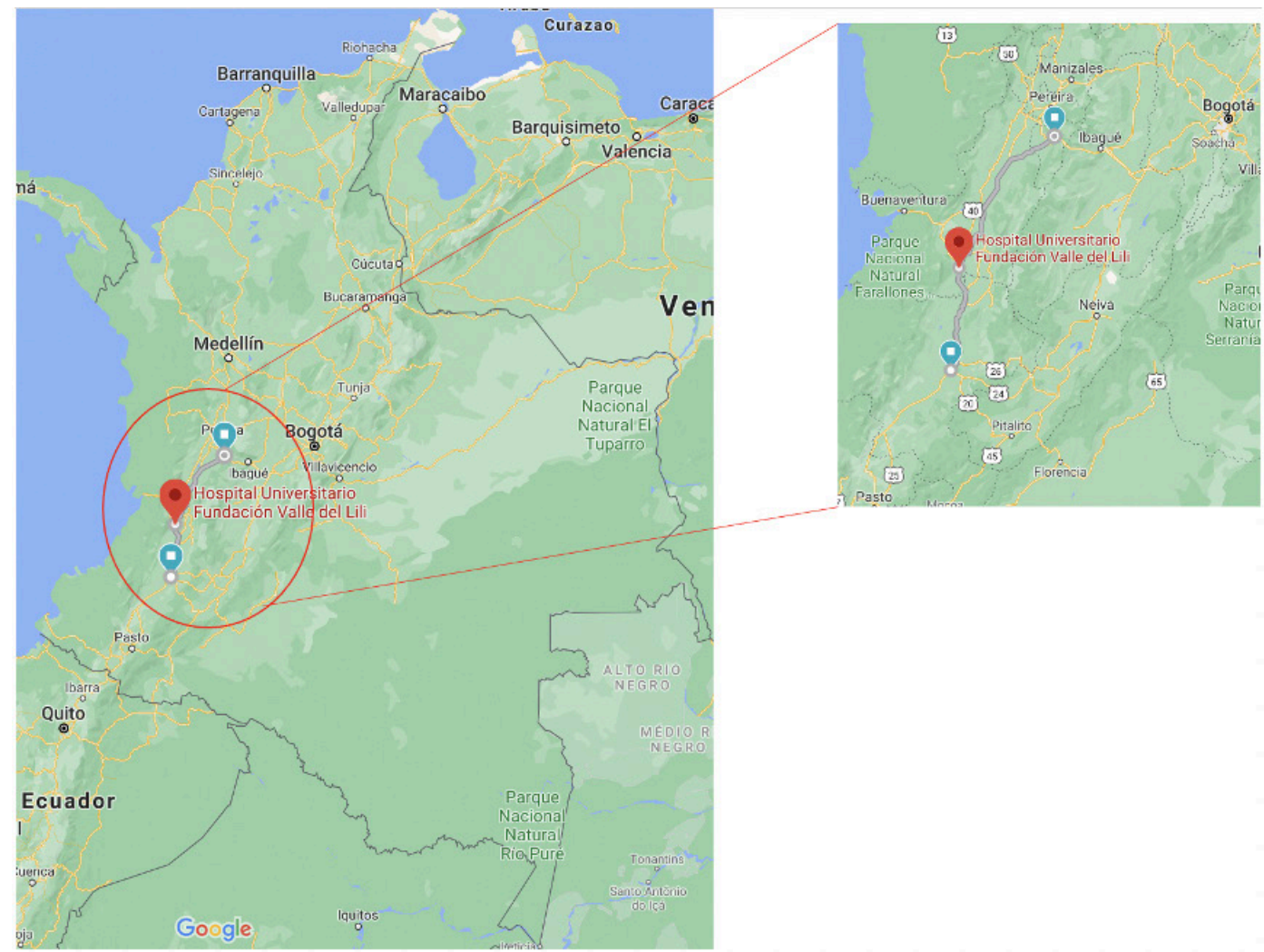

$1 a$

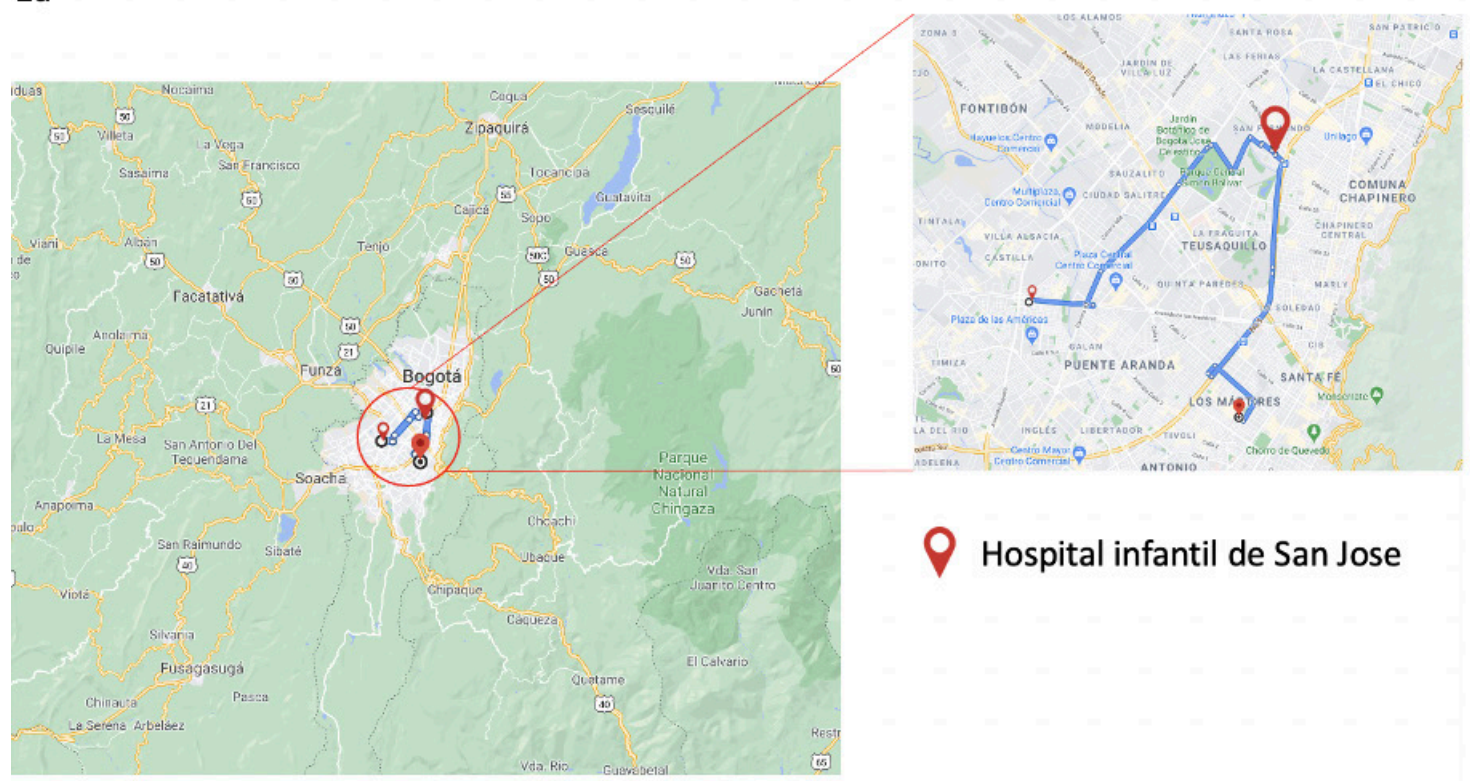

$1 b$ 


\section{Resultados}

De acuerdo con el Global Burden Of Disease, la incidencia de ictus isquémico en Colombia fue de 25.359 casos en el año 2019, generando 8.295 muertes (6.266-10.477) y un número absoluto de 137.162 DALYs (Disability Adjusted Life Years) (11.328 - 166.980)? A nivel nacional, solo 32 hospitales distribuidos en 19 ciudades participan en el Registry of Stroke Care Quality (RES-Q), que durante el año 2019 incluyó 3352 pacientes con ictus de los cuales 2191 correspondieron a ictus isquémico, $20.5 \%$ recibió terapia de reperfusión y de estos, en 7 casos $(0.32 \%)$ se aplicó la estrategia "Drip and ship". Con los datos anteriormente consignados podemos estimar que el registro RES-Q, recoge aproximadamente un $8,6 \%$ del total de casos de ictus isquémico en Colombia.

En el año 2020 se registraron en el RES-Q 4200 pacientes con ictus, de los cuales 3017 correspondian a ictus isquémico (71.83\%), de estos pacientes el 18.93\% se sometió a terapia de reperfusión, y de estos el $0.3 \%$ (9 casos) se hicieron bajo la estrategia de "Drip and ship". En el primer cuartil de 2021 se han registrado 1042 ictus, de los cuales 746 son de etiología isquémica, de estos el $23.59 \%$ se sometió a terapia de reperfusión, siendo el $0.54 \%$ (4 casos) de los casos realizada con la estrategia de "Drip and ship".

De los 20 casos que se reportaron en el registro RES-Q que recibieron "Drip and ship" durante el periodo de tiempo seleccionado, solo se incluyeron los casos de los centros que respondieron a la convocatoria realizada a través de la Asociación Colombiana de Neurología: tres casos del 2019, dos del 2020 y uno del 2021.

La mediana de edad fue de 63.5 años (RIC, 54.5$72.5)$, todos presentaban un mRs previo favorable (0-2) y el sexo femenino representó el $84 \%$ de los casos. Dentro de las comorbilidades, dos pacientes tenían fibrilación auricular (FA) sin anticoagulación y otro de los pacientes debutó con FA, una paciente tenía hipertensión arterial, otro paciente con antecedente de ictus y una paciente sin comorbilidades previas. Dos de los pacientes fueron tratados en un centro sub-especializado de ictus en Cali, de los cuales uno fue remitido de Armenia (192 km, tiempo de traslado $175 \mathrm{~min}$ ) con prenotificación y 1 caso desde Popayán (141 km, tiempo de traslado de $182 \mathrm{~min}$ ) sin prenotificación (Figura 1). En Bogotá, los 4 casos fueron remitidos de centros primarios de la misma ciudad a una distancia promedio de $9.5 \mathrm{Km}$ y un tiempo de $61.25 \mathrm{~min}$. La mediana de tiempo desde el inicio de los síntomas hasta la llegada al centro sub-especializado (tiempo de ventana) fue de 60 minutos (RIC: $30-180$ ) sin embargo, en el $16.66 \%$ de los casos el tiempo de ventana fue desconocido y fue superior en los pacientes provenientes de otras ciudades. La mediana de puntaje NIHSS de ingreso al centro primario fue de 16.5 puntos, compatible con oclusión pro- ximal y la mediana de ASPECTS en la imagen de remisión fue de 10, a tres pacientes se les realizó TC simple, a 2 casos de angiografía por tomografía computarizada (CTA) y al restante RMN con DWI/FLAIR. La mediana de tiempo puerta-aguja fue de 75 minutos (52.5-97.5); el $75 \%$ de los pacientes fueron trasladados dentro de la misma ciudad, en este grupo el tiempo puerta aguja fue $\leq$ a 60 minutos. El tiempo door-in door-out fue superior en pacientes provenientes de otras ciudades con una mediana de 237.5 min en comparación con los pacientes dentro de la misma ciudad con mediana de $160 \mathrm{~min}$. Al llegar al centro sub-especializado a todos los pacientes se les realizó nuevamente neuroimágenes; a tres pacientes se les realizó tomografía computarizada con perfusión (CTP), a dos se le realizó TC + CTA y solo a uno se le realizó TC de cráneo simple, la mediana de la escala ASPECTS fue de 7 puntos, evidenciando la progresión del infarto. Al $83.3 \%$ se les realizó trombectomía mecánica, el caso \# 2 al llegar al centro sub-especializado se realizó imagen con ASPECTS 3 puntos descartando manejo con TM. De los pacientes llevados a trombectomía se obtuvo un TICI score de 3 en $66.66 \%$ casos y el restante, TICI 2 c. La etiología del ictus isquémico fue cardioembólico en 5 de los 6 pacientes. Los días de estancia hospitalaria en el centro de alta complejidad en Cali fue de 4.5 días mientras que en el centro de Bogotá fue de 6. Finalmente, el $50 \%$ de los pacientes presentaron un $\mathrm{mRs}$ favorable (0-2) al egreso y a los 3 meses fue favorable en el $84 \%$, la mayoría de estos pacientes pertenecientes al grupo que se desplazó dentro de la misma ciudad.

\section{Discusión}

La estrategia Drip and Ship ha demostrado su eficacia en diferentes estudios. ${ }^{9} 10$ En la revisión narrativa de la literatura no encontramos reportes de trombolisis en centro primario y remisión a trombectomía en Colombia, sin embargo, usando datos de retroalimentación del registro RES-Q solamente se registró "drip and ship" en el $0.32 \%$ de los casos en nuestro país. En Latinoamérica se ha reportado su práctica en Chile en el registro de un programa de tele-trombolisis donde se remitió al 7\% de los pacientes que recibieron trombolisis para $\mathrm{TM}^{10}$

En Brasil un estudio que incluyó 300 pacientes, estudió la eficacia de la trombectomía en 12 hospitales públicos en pacientes con oclusión intracraneal proximal de la circulación anterior que podía tratarse dentro de las 8 horas del inicio de los síntomas, se aleatorizaron en razón 1:1 para recibir manejo estándar con alteplasa vs manejo estándar versus TM y se midieron los desenlaces con la mRS. De los 300 pacientes, aproximadamente el 70\% recibió alteplasa en ambos grupos. El estudio se detuvo de manera prematura por eficacia temprana cuando 221 de 690 pacientes se aleatorizaron (111 al grupo de trombectomía y 110 al grupo control). El odds ratio (OR) para 
una mejor distribución de las puntuaciones en la mRS a los 90 días fue de 2.28 (intervalo de confianza [IC] de 95\% 1.41-3.69; $\mathrm{P}=0.001$ ), favoreciendo la trombectomía. De los pacientes con mRS 0-2 el 35.1\% recibieron terapia con TM, el 20\% fueron asignados al grupo control. La hemorragia intracraneal asintomática sucedió en $51.4 \%$ de los casos del grupo de trombectomía y $24.5 \%$ en el grupo control.1.

En Estados Unidos la estrategia Drip and Ship es común, se emplea en 1 de cada 4 pacientes incluyendo casos sin oclusión de vaso proximal que son trombolizados y son trasladados a centros sub-especializados para cuidados especiales, y aquellos que se remiten para TM.12

Los dos centros sub-especializados a los que ingresaron los pacientes son hospitales universitarios, con un programa de ictus establecido y están ubicados en dos de las principales ciudades del país. La prenotificación de los pacientes no se realizó en todos los casos y en aquellos que se realizó, se hizo de manera informal entre los médicos tratantes de los pacientes.

En nuestro grupo de pacientes, el $83.3 \%$ de los casos fueron de etiología cardioembólica. Con una proporción mayor a lo reportado en un subanálisis del estudio $\mathrm{Mr}$ CLEAN donde el 33\% correspondió a cardioembolismo y $49 \%$ con etiología desconocida, ${ }^{11} 2$ casos tenían antecedente conocido de fibrilación auricular y no estaban recibiendo anticoagulantes, por motivos desconocidos, un caso se diagnosticó con FA de novo. En el caso \#5 no se logró establecer la etiología del ictus a pesar de realizar estudios de extensión. Los pacientes que tuvieron que ser trasladados a una ciudad diferente a su origen, tuvieron que desplazarse una mayor distancia y durante un mayor tiempo. La poca disponibilidad de centros de $\mathrm{TM}$ en varias regiones del país dificulta el acceso de un porcentaje importante de la población de nuestro país a tratamientos combinados (TE y TM).

En nuestra serie, 3 pacientes tuvieron un desenlace favorable al egreso definido como mRs (0-2), 2 pacientes presentaron mRS moderado de 3 y un paciente falleció anotando que tenía un infarto establecido con ASPECTS 3 a la llegada al centro sub-especializado y no fue candidato a TM. Previamente se ha publicado que los desenlaces de los pacientes con ictus sometidos a la estrategia de Drip and Ship son mejores. Un estudio evidenció que estos pacientes tenían NIHSS más bajos, menor mortalidad intrahospitalaria y menos hemorragia intracraneal sintomática comparados con los pacientes que fueron trasladados directamente a centros primarios. ${ }^{12}$ En el estudio citado el tamaño de la muestra fue de 44.667 por lo que no es comparable con nuestra serie de casos.

En nuestro país existen varios retos que dificultan la atención de los pacientes con ictus. Existe un flujo escaso de información entre el centro primario y el centro subespecializado el cual podría mejorarse mediante la noti- ficación sistemática del traslado de los pacientes pues un retraso de 20 minutos en el tratamiento puede reducir hasta en un $20 \%$ la probabilidad de un desenlace favorable. ${ }^{13}$ La falta de implementación y uso rutinario de escalas que permiten sospechar oclusión proximal en la atención pre hospitalaria o centros primarios de atención, como la escala RACE y FAST-ED, han demostrado identificar de forma exitosa obstrucciones arteriales proximales en el contexto prehospitalario. ${ }^{14,15}$ Además, puede existir una disparidad marcada en la atención prestada a los pacientes con ictus provenientes de países de bajos y medianos recursos como el nuestro principalmente por infraestructura insuficiente, donde cobran gran importancia las unidades de ictus. ${ }^{16}$

Desde 2011 hay reportes de casos exitosos en el que manejan de forma rutinaria el "Drip and Ship", por lo general mediante el uso de tecnologías como teleconsulta. ${ }^{17}$ Estas estrategias pueden mejorar los desenlaces de los pacientes con ictus en zonas rurales, aumentando el uso de rt-PA. Dada la evidencia científica que soporta este tipo de intervenciones en beneficio de los pacientes y la costo efectividad de la trombolisis para Colombia ${ }^{18}$ se deben fortalecer las redes nacionales de ictus e implementar en el sistema de salud la atención del ictus como una emergencia médica que permita acceso a la atención oportuna, implementación de centros primarios y subespecializados de atención para ictus así como ampliar el uso de telemedicina y garantizar un transporte óptimo ${ }^{19}$ pueden ofrecer desenlaces favorables en la población. La atención integral a los pacientes plantea un reto enorme para el Sistema de Salud colombiano dadas las condiciones geográficas, las múltiples barreras de acceso a la red prestadora de servicios para ciertas poblaciones, y la ausencia de una red de atención para el paciente con ictus isquémico en Colombia puesto que en la mayoría de los casos, no se cuenta con la infraestructura especializada ni personal calificado disponible 24 horas. $^{20}$

Es importante mencionar que dentro de las limitaciones de este estudio se encuentra la recolección de datos de forma retrospectiva, mediante invitación o revisión de registros, posiblemente excluyendo la participación de centros de práctica privada en la serie, lo cual no permitió recolectar información de los pacientes tratados con esta estrategia durante el periodo de tiempo descrito. Además, este registro incluye un porcentaje reducido del total de casos en el país, un estimado del 8,6\% de los casos de ictus nacionales, donde además se estima que hay un subregistro de los casos, especialmente en los hospitales sin un programa de atención de enfermedades vasculares cerebrales.

En conclusión, a partir de la experiencia descrita en otros países en la implementación de la estrategia Drip and Ship, aumenta el porcentaje de pacientes que reciben terapias de reperfusión. En Colombia, el chance de ser 
sometido a Drip and Ship es casi anecdótico, sin embargo, se podría considerar el desarrollo de una estrategia para su implementación. Para ello, es importante mejorar el flujo de información entre el centro primario y el centro sub-especializado de forma que se reduzcan los tiempos de manejo en centro primario y de traslado. Se requieren estrategias de planeación para la creación de lineamientos nacionales, departamentales y regionales que permitan una adecuada estructuración de la ruta de atención para la creación de la red de ictus. Además fortalecer el uso de registros regionales y nacionales de información para evaluar la calidad de atención y el impacto de políticas públicas de salud como lo han logrado otros países del continente.

\section{Referencias}

1. Ferri, C. P., Schoenborn, C., Kalra, L., et al. Prevalence of stroke and related burden among older people living in Latin America, India and China. Journal of Neurology, Neurosurgery \& Psychiatry. 2011; 82(10), 1074-1082. https://doi.org/10.1136/ jnnp.2010.234153

2. Ishihara, H., Oka, F., Oku, T., et al. Safety and Time Course of Drip-and-Ship in Treatment of Acute Ischemic Stroke. Journal of Stroke and Cerebrovascular Diseases. 2017; 26(11), 2477-2481. https://doi. org/10.1016/j.jstrokecerebrovasdis.2017.03.008

3. Bayona-Ortiz H, Useche J, Yanez N, Velasco S. Availability of stroke units in Colombia. Lancet Neurol. 2019;18(11):988. https://doi.org/10.1016/ S1474-4422(19)30332-1

4. Goyal, M., Menon, B. K., van Zwam, W. H., et al. Endovascular thrombectomy after large-vessel ischaemic stroke: a meta-analysis of individual patient data from five randomised trials. The Lancet. 2016; 387(10029), 1723-1731. https://doi.org/10.1016/ s0140-6736(16)00163-x

5. Powers, W., Rabinstein, A., Chair, V., Ackerson, T., \& Adeoye, O. (2019). Correction to: Guidelines for the Early Management of Patients With Acute Ischemic Stroke: 2019 Update to the 2018 Guidelines for the Early Management of Acute Ischemic Stroke: A Guideline for Healthcare Professionals From the American Heart Association/American Stroke Association. Stroke, 50(12). doi: 10.1161/ str.0000000000000215

6. Müller-Barna, P., Hubert, G. J., Boy, S., et al. TeleStroke Units Serving as a Model of Care in Rural Areas. Stroke. 2014; 45(9), 2739-2744. https://doi. org/10.1161/strokeaha.114.006141

7. Vizhub.healthdata.org. 2021. GBD Compare | IHME Viz Hub. [online] Available at: <https://vizhub. healthdata.org/gbd-compare/> [Accessed 09 September 2021].

8. Registry for Stroke Care Quality (RES-Q). National reports 2019. Disponible en: https://www.qualityregistry.eu/data/login/?next=/data/dashboard_home/

9. Martin-Schild, S., Morales, M. M., Khaja, A. M., et al. Is the Drip-and-Ship Approach to Delivering Thrombolysis for Acute Ischemic Stroke Safe? The Journal of Emergency Medicine. 2011; 41(2), 135141. https://doi.org/10.1016/j.jemermed.2008.10.018

10. Mansilla E, Mazzon E, Cárcamo D, Jurado F, et al. Telestroke en Chile: resultados de 1 año de experiencia de la Unidad de TeleACV del Servicio de Salud Metropolitano Sur en 7 hospitales ejecutores. RevistamédicadeChile.2019;147(9):1107-1113.http:// dx.doi.org/10.4067/s0034-98872019000901107

11. Martins, S., Mont'Alverne, F., Rebello, et al. Thrombectomy for Stroke in the Public Health Care System of Brazil. New England Journal of Medicine. 2020; 382(24), pp.2316-2326. https://doi.org/10.1056/ NEJMoa2000120

12. Sheth, K. N., Smith, E. E., Grau-Sepulveda, M. V., et al. Drip and Ship Thrombolytic Therapy for Acute Ischemic Stroke. Stroke. 2015; 46(3), 732-739. https://doi.org/10.1161/strokeaha.114.007506

13. Kim, Y. S., Kim, B. J., Noh, et al. Distal versus Proximal Middle Cerebral Artery Occlusion: Different Mechanisms. Cerebrovascular Diseases. 2019; 1-7. https://doi.org/10.1159/000500947

14. Tekle, W. G., Chaudhry, S. A., Hassan, et al. Dripand-Ship Thrombolytic Treatment Paradigm Among Acute Ischemic Stroke Patients in the United States. Stroke. 2012; 43(7), 1971-1974. https://doi. org/10.1161/strokeaha.112.657817

15. Kim, S. K., Lee, S. Y., Bae, H. J., Lee, et al. Pre-hospital notification reduced the door-to-needle time for iv t-PA in acute ischaemic stroke. European Journal of Neurology. 2009; 16(12), 1331-1335. https://doi. org $/ 10.1111 / j .1468-1331.2009 .02762 . x$

16. Pérez de la Ossa, N., Carrera, D., Gorchs, M., et al. Design and Validation of a Prehospital Stroke Scale to Predict Large Arterial Occlusion: The Rapid Arterial Occlusion Evaluation Scale. Stroke. 2013; 45(1), 87-91. https://doi.org/10.1161/strokeaha.113.003071

17. Lima, F. O., Silva, G. S., Furie, K. L., et al. Field Assessment Stroke Triage for Emergency Destination. Stroke. 2016; 47(8), 1997-2002. https://doi. org/10.1161/strokeaha.116.013301

18. Chimatiro, G. L., \& Rhoda, A. J. Scoping review of acute stroke care management and rehabilitation in low and middle-income countries. BMC Health Services Research. 2019; 19(1). https://doi.org/10.1186/ s12913-019-4654-4

19. Gallerini, S., Marsili, L., Groccia, V., et al. Appropriateness, safety, and effectiveness of "drip and ship" teleconsultation model in Southeastern Tuscany: a feasibility study. Neurological Sciences. 2020. https://doi.org/10.1007/s10072-020-04446-x

20. Moreno E, Rodríguez J, Bayona-Ortiz H. Trombó- 
lisis endovenosa como tratamiento del ACV isquémico agudo en Colombia: una revisión sistemática de la literatura. Acta Neurológica Colomb [Internet]. 2019 Sep 18 [cited 2020 Sep 24];35(3):156-66. https://doi.org/10.22379/24224022262

Conflictos de interés: Los autores declaran que no tienen conflictos de interés o relaciones personales que puedan influenciar el trabajo reportado en este manuscrito. Pablo Amaya ha recibido honorarios como conferencista de Boehringer Ingelheim, Abbott Structural Heart y Boston Scientific. Angel Corredor ha recibido honorarios como conferencista de Boehringer Ingelheim. 